\title{
Embodied perspectives on behavioral cognitive enhancement
}

\author{
Zona, C. I. ${ }^{1}$, Raab, M. ${ }^{2,3}$, \& Fischer, M. H. ${ }^{1}$ \\ 1 - University of Potsdam, Germany \\ 2 - German Sport University Cologne, Germany \\ 3 - London South Bank University, UK
}

Length: 10,812 words (excluding 112 references); 3 Figures, no Tables

Send correspondence to:

Prof. Martin H. Fischer, PhD

Department of Psychology, University of Potsdam

Karl-Liebknecht-Strasse 24-25 House 14

D-14476 Potsdam OT Golm, Germany

Email: martinf@uni-potsdam.de 


\begin{abstract}
Recent debates about strategies to enhance human cognition concerned mostly pharmacological, environmental and genetic factors, as well as computerized cognitive training paradigms targeting healthy populations. We offer a new perspective on behavioral cognitive enhancement, arguing that embodied cognition represents a productive framework to explain results and to inform new studies aimed at enhancing cognition. Understanding cognitive mechanisms and their time-course through an embodied perspective contributes to our knowledge of brain functioning and its potential. We review two domains: a) physical exercise and b) embodied learning. For each domain, we summarize experimental evidence according to the level of embodiment of the knowledge representations targeted by interventions (i.e., situatedness, embodiment proper, grounding). Future research should integrate embodiment and cognitive enhancement in training paradigms focused on joint cognitive and physical tasks.
\end{abstract}

(128 words)

\title{
Keywords:
}

cognitive enhancement, cognitive training, embodied cognition, embodied learning, physical exercise. 


\section{Embodied perspectives on behavioral cognitive enhancement}

All scientific approaches to human cognition have traditionally emphasized performance and its enhancement: Behaviorists have looked at learning rates and optimized operant behaviours; cognitive psychologists have analyzed data-limited and resource-limited performance in more or less productive dual-task combinations (e.g., Norman \& Bobrow, 1975); mental chronometry has been used to measure the speed of cognitive processes and their associated accuracy, thereby inferring mental constructs such as executive functions (e.g., Miyake \& Friedman, 2012), working memory (Baddeley, 1992) and fluid intelligence (Jaeggi, Buschkuehl, Jonides, \& Perrig, 2008). Faster task completion has been equated with more efficient use of these hypothetical constructs and their associated mental resources, resulting in congruency benefits in the short term and in enhanced cognition in the longer term (Lachman, Lachmann, \& Butterfield, 1979). Recently such benefits in cognitive enhancement and embodied cognition have been labeled either as acute versus chronic enhancement effects (McMorris, 2016) or as online versus offline embodied effects (Schütz-Bosbach \& Prinz, 2007). Both such differentiations show that the effects on cognition are conceptualized on an underspecified time line; ultimately they shape cognition throughout our entire life-span (Löffler, Raab, \& Cañal-Bruland, 2016).

The recent debate about strategies for enhancing human cognitive abilities has concerned mostly pharmacological, environmental, or genetic factors (Dubljević, Knafo \& Venero, 2015) and cognitive training interventions in healthy populations (Klingberg, 2010; Colzato, 2017). Outcomes are typically measures of training-induced transfer (e.g., Söderqvist \& Nutley, 2017), based on the notion that systematically engaging a specific cognitive skill will enhance performance in untrained tasks supported by that 
skill. This line of research has received increasing attention; however, low comparability, statistical shortcomings and low ecological validity have limited its impact on the field (Moreau, Kirk, \& Waldie, 2016; Söderqvist \& Nutley, 2017; Cremen \& Carson, 2017).

The embodied approach to human cognition offers a fresh perspective on cognitive enhancement. Under this account, sensorimotor and proprioceptive features of training experiences are obligatorily encoded in the same neural structures underlying cognition, thereby generating multi-modal knowledge representations that support all cognitive activities (e.g., Barsalou, 2008; Coello \& Fischer, 2016; Fischer \& Coello, 2016). All previous experiences are thereby integrated to generate adequate cognitive strategies aimed at both understanding and acting flexibly upon the environment. These cognitive strategies can be understood as jointly determined by three layers (or parameters) of knowledge representation (see Figure 1): first, by physical properties of the environment and the organism that evolved in this environment (grounding); secondly, by an organism's bodily structure and its resulting sensori-motor repertoire (embodiment proper); and thirdly by its position within the immediate environment and the currently required behaviour (situatedness; cf. Pezzulo, Barsalou, Cangelosi, Fischer, McRae, \& Spivey, 2011; Fischer, 2012). The key cognitive mechanism that relies on these hierarchically related knowledge representations is simulation, i.e., the multimodal partial re-enactment of experiences in the respective brain systems; these simulations have been shown to support diverse cognitive abilities such as motor learning, social understanding, language comprehension and even mental arithmetic (e.g., Glenberg \& Gallese, 2012; Fischer \& Shaki, 2014, 2018; Coello \& Fischer, 2016; Fischer \& Coello, 2016). 
Insert Figure 1 about here

While the exact theoretical framing and neural implementation of simulation mechanisms is under debate (e.g., Pulvermüller, 2013; Topolinski \& Strack, 2015; Gentsch, Weber, Synofzik, Vosgerau, \& Schütz-Bosbach, 2016), our aim in this contribution is to summarize mainly behavioral findings of research in cognitive enhancement from an embodied cognition perspective, to argue that it represents a productive theoretical framework for interpreting previous results, and to experimentally address outstanding questions. In this review, we refer to behavioral strategies to cognitive enhancement in order to designate voluntary behaviours which contribute to maximizing cognitive performance. Although some of the approaches reviewed require instruments other than the body to aid such behavior, technology is used non-invasively by all approaches considered in this review. Thus, studies of cognitive enhancement due to pharmacological means and invasive techniques such as brain stimulation are excluded here (but see e.g., Colzato, 2017).

The embodied approach has received converging support and demonstrated the applicability of its parameters by encompassing experimental results from several research paradigms aimed at enhancing human cognition. These empirical findings support the view that cognition can be enhanced by interventions targeting the optimal integration of sensory, motor, interoceptive and proprioceptive states to master cognitive 
challenges. We aim to show how previous findings from behavioral studies of cognitive enhancement can be interpreted in terms of their relationship with parameters relevant to embodied cognition. The effects of engaging participants in integrated physical and cognitive challenges are proposed as a common denominator of successful interventions and as an important factor underlying individual differences in adaptive behavior and traits. Specific evidence for far-reaching transfer - i.e., highly generalizable across disparate tasks - has been obtained in studies investigating cognitive advantages induced by physical exercise and embodied learning. These are the two cognitive domains we will review below.

The choice of these domains is motivated by the nature of their connection to cognitive science research, which can be interpreted as being mediated by embodied parameters. On the other hand, we will not consider in this review domains such as sleep and nutrition, which have also been demonstrated to contribute substantially to better cognitive performance, as well as physical and mental health (Gómez-Pinilla, 2008; Potkin et al., 2012). Effects of such factors are not directly linked to specific cognitive capacities, but rather diffused over brain functioning at large. For instance, sleep has been shown to enhance a wide range of cognitive abilities, most notably memory. Recent evidence from animal and human studies has found large overlaps of neurotransmitters and neuromodulators involved in sleep and memory consolidation not only in the neurobehavioral domain, but also in the formation of long-term memories about the body's past immunological responses (Rasch \& Born, 2012). Furthermore, recent evidence from mice studies showed that sleep plays a fundamental role in brain function in that it allows the removal of neurotoxic waste from the brain through enhanced exchange of cerebrospinal and interstitial fluid (e.g., Xie et al., 2013). Thus, it 
seems that the causal links between sleep and cognitive enhancement are rooted in the biological bases of sleep in deep and complex ways, the analysis of which is beyond the scope of this review. In contrast, the behavioral domains considered here have demonstrated their suitability at enhancing specific cognitive capacities at varying timescales and expertise levels by means of empirical paradigms that are informed by the embodied cognition framework.

\section{Physical exercise}

Physical exercise has been demonstrated to promote metabolic, anatomical and chemical changes supporting optimal brain function. In particular, aerobic exercise, defined as sustained physical exercise at a moderate pace over a minimum of 20 minutes, has been found to improve general health markers - such as lowering blood pressure and resting heart-rate levels - and to promote neurovascularization and angiogenesis (Dietz, 2013). Moreover, physical exercise triggers the release of neurotransmitters such as dopamine and norepinephrine, as well as brain-derived neurotrophic factor (Moreau, 2015). This protein supports a wide range of neurophysiological changes such as neurogenesis and synaptogenesis, and contributes crucially to stem cell and neuronal survival, as well as preventing neuron degeneration, all factors suggested to contribute to individual differences in cognitive capabilities (Daamen \& Raab, 2012).

Due to low comparability between studies, however, transfer of different types of physical exercise onto specific cognitive constructs beyond physiological measures has not been clearly established (Dietz, 2013). Indeed, cognitive and physical challenges have typically been addressed separately, whereas recent research proposing an integrative approach has aimed at merging both cognitive and physical demands into 
one activity (McMorris, 2016). Within the embodied cognition framework, at least four distinct experimental paradigms have been deployed to determine the effectiveness of simultaneous manipulations of both physical and cognitive tasks for cognitive enhancement. Here, we review evidence in support of this claim according to its location on a timeline spanning from immediate to long-lasting beneficial effects on cognitive and motor performance. First, we consider manipulations of physical effort, whose effects are already evident immediately after the intervention. This approach is based on the idea that simultaneous physical and cognitive demands will improve performance due to the higher competition for physiological resources among various organs, which will challenge the brain to function more efficiently (Moreau, 2015). In a second line of research, mental imagery has been investigated as a potential cognitive mechanism underlying beneficial effects evident on motor coordination and athletic and artistic performance within hours after the interventions.

Thirdly, evidence for longer-lasting cognitive benefits was provided by motor training paradigms, specifically designed to integrate cognitive and motor demands and target the integration of proprioceptive and sensorimotor information to successfully complete an interactive cognitive challenge. Fourthly and finally, the embodied cognition approach has been used to explain learning and practising of optimal motor behaviour in terms of an agent's acquisition of motor expertise, which is correlated to faster and better performance due to year-long training experience.

We next review several examples of these four approaches, so as to illustrate the close connection between cognitive enhancement and embodied cognition. To foreshadow our conclusion, the findings reviewed in this section point to the relevance of embodied parameters to enhancing both on-line and off-line cognitive processing at 
varying timescales, as illustrated in Figure 2.

Insert Figure 2 about here

\subsection{Physical effort}

In the first approach, physical and cognitive demands, although simultaneously occurring, are unrelated in terms of their meaning and function, and physical exercise is merely a means to increase levels of bodily arousal and/or perceived physical effort. Due to the fact that manipulations crucially involve situated parameters, the observed effects on cognition are limited to a relatively narrow time-window. However, if the conditions experienced by the agent are rather due to its embodiment proper (e.g., depending on individual levels of physical fitness), similar effects can be hypothesized to last much longer. Typical procedures of this paradigm involve a conceptuometaphorical transposition of effort (often in terms of weight manipulations) onto the cognitive domain, such that bodily cues bias the judgement of perceptual stimuli (e.g., a hill looks steeper if participants are wearing a heavy backpack; Bhalla \& Proffitt, 1999, but see Woods, Philbeck, \& Danoff, 2009, for an alternative explanation). Consistent with a fundamental insight of embodied cognition, this procedure demonstrates that somatosensory cues can bias cognition and metacognition.

When participants complete a cognitive task with or without sustaining a parallel physical effort, manipulations of weight have shown different effect patterns. On the one hand, physical effort leads participants to overestimate the difficulty of their 
current cognitive task, decreasing performance and confidence ratings for successive retrieval of learned items (e.g., carrying a heavy vs. a light backpack). On the other hand, physical effort has also been shown to increase performance and metacognitive judgement of learning, ascribed to the fact that weight cues might lead participants to allocate more attention to "heavier" items (Alban \& Kelley, 2013). For instance, in a recent study, participants were asked to memorize easy and difficult nouns while half of them wore a backpack loaded with $15 \%$ of their bodyweight (Skulmowski \& Rey, 2017). Physical effort enhanced learning for both word categories, leading the authors to conclude that perceived effort may help directing attention towards targeted stimuli, for which learning is then improved. Moreover, an interaction of physical effort and cognitive effort (i.e., word difficulty) was evident in confidence ratings, in that physical effort improved learning confidence for easy words while only little effect of weight manipulation on confidence about difficult words was shown. This result was interpreted by the authors as supporting evidence for the dual-process model of cognition, which posits that (perceived) task difficulty should activate a more thorough mode of cognitive processing (Skulmowski \& Rey, 2017).

This line of research generally supports the notion that principles of embodied cognition hold when physical effort is manipulated for cognitive enhancement. However, a methodological flaw might be that insights from conceptual metaphor theory (e.g., Lakoff \& Johnson, 2003) are applied to interpret the results in terms of conceptual-system relationships. While metaphoric links between concepts need to fulfil intrinsic semantic constraints, they are otherwise characterized by a high degree of flexibility across different contexts and uses. For instance, the "weight" metaphor has been suggested to evoke the importance of the cognitive task's content, and thus to 
enhance attention outcomes, cognitive effort and performance (Alban \& Kelley, 2013; Jostmann et al., 2009). Other research, however, has suggested that weight cues can also be interpreted as a metaphorical cue for the concept "burden", and these studies indeed observed the expected deleterious effect of weight manipulations on cognitive performance (Kouchaki, Gino, \& Jami, 2014; Slepian, Masicampo, Toosi, \& Ambady, 2012). Thus, it seems that these interpretational options need to be thoroughly controlled for by future research, so as to avoid interpreting empirical findings in a confirmatory fashion. Furthermore, dose-response relations between physical effort and cognitive performance are not well understood. For instance, an individualized 60\% $\mathrm{VO}^{2}$-max exercise may have positive effects on a perceptual-cognitive task whereas a 90\% exercise may not (Drid, Majstorovic \& Drapsin, 2010).

\subsection{Mental imagery}

The evidence reviewed so far highlights the importance of incorporating an embodied, physical component into otherwise computerized cognitive training paradigms and has revealed the influence of signal integration on cognitive abilities. In research dedicated to mental imagery, increasing evidence points to an opposite, complementary facet of the relationship between cognitive and motor processes. Imagery is generally defined as "a perception-like process in the absence of any external stimulus" (Munzert, Lorey, \& Zentgraf, 2009, p. 307) and, in embodied accounts, is often characterized as a particular kind of embodied simulation. Simulation typically recreates an integrated multisensory account of perceptual experiences, but whereas embodied simulation happens automatically in the presence of external stimuli, mental imagery needs to be deliberately generated and maintained over time. Furthermore, research on imagery has distinguished between perceptual, motor and exercise imagery. 
Whereas perceptual - or visual - imagery consists in mentally visualizing features of experience from a first- or a third-person perspective, motor - or kinesthetic - imagery simulates kinesthetic features of motor behavior and their (predicted) proprioceptive consequences, typically from a first-person perspective. Exercise imagery, on the other hand, simulates outcomes and processes particular to athletic and exercise behaviour, with the goal of enhancing performance through an increase in feelings of self-efficacy and motivation (Kalicinski \& Lobinger, 2013). Here, the focus will lie mainly on kinesthetic imagery since we believe it represents strong evidence for the grounding of simulated and executed behaviour upon overlapping neural structures, as well as providing a powerful example of the mutual influence of cognitive and motor mechanisms, reflected in transfer between overt and covert performance (see Munzert et al., 2009, for a review and conceptual clarifications of mental and motor/kinesthetic imagery).

In support of embodied theories of cognition, increased neural activation during motor imagery is typically found in the premotor and primary motor cortices, as well as in the basal ganglia and in subcortical areas of the cerebellum (Jeannerod, 2006; Munzert et al., 2009). These activation patterns overlap largely with neural circuitry which is involved in motor execution, as well as motor observation, action planning and even action-related language processing (Pulvermüller, 2005; 2013). The same cortical areas have been identified as the core structures constituting the mirror-neuron system in humans (Rizzolatti \& Craighero, 2004), a cortical network found to support action observation and suggested to facilitate subsequent learning. Performance enhancement through mental practice builds on the knowledge that, since movement simulation and execution engage the same neurocognitive mechanisms in the motor system, 
strengthening the connections in neural networks subserving action through its mental simulation improves overt performance (Gentili, Papaxanthis, \& Pozzo, 2006). Thus, not only do embodied accounts of cognition provide a convincing framework for the observed effectiveness of mental training for motor and sport performance, but also these results can add to the knowledge about the nature of motor representations in the brain and their relationship with more abstract cognitive processes, such as mental arithmetic (cf. Werner \& Raab, 2013; Fischer \& Shaki, 2014, 2018).

In EEG, EMG and kinematics studies, the extent to which motor knowledge and motor learning of optimal performance is transferred to different situations is often investigated at different levels of analysis. One example is the generalization of goaldirected movement from one effector to another (Gentili et al., 2006); another example is the modulation of activation patterns during action observation and motor imagery as a function of the degree of expertise in complex motor activity (Guillot, Lebon, Rouffet, Champeley, Doyon, \& Collet, 2007; see also Di Nota, Chartrand, Levkov, MontefuscoSiegmund, \& DeSouza, 2017). We discuss these examples of motor imagery research in some detail to illustrate and support our argument.

In the study by Gentili and colleagues (2006), 40 participants were first made to complete a motor training session. At a desk, a series of 12 target numbers was displayed by a mechanical device in two parallel rows in front of the participant. The goal of the motor task was to complete one of two paths, marked either with black or white numbers, by pointing at the correct upcoming target, which was each time indicated in either colour on the edge of the currently attended tag. The two paths, although symmetrical, posed different constraints on arm joints and motor dynamics, in particular as a consequence of gravity and of the inertial anisotropy of the arm - i.e., the 
limb dynamics by which the inertial resistance is determined by the direction of the arm movement. During the pointing task, participants were to integrate predictions from external (gravity) and internal (anisotropy) dynamics and rapidly use them to inform optimal motor behaviour. In a pre-training session, participants practiced the task, and baseline measurements of kinematics parameters and EMG recordings served as reference performance to assess post-intervention improvement within subjects. Following this step, participants were randomly allocated to one of four groups: physical training, motor imagery training, an active control group, or a passive control group. In the first condition, participants practiced by actually performing the movements required for the task, in much the same way as during the pre-training assessment; in contrast, participants in the motor imagery training were only supposed to create a simulation of themselves performing the movements in the most accurate way, but without overt motor execution (this latter condition resembles the simulation process postulated by embodied cognition). The passive control group did not receive any training, while the active control group's training instructed participants to only perform eye movements towards the targets, without generating kinesthetic imagery. This control condition is of great relevance to determine the extent to which improvements shown in the target group are due to kinesthetic imagery proper, and distinguishable from those due to saccadic motion typically reported during the generation of kinesthetic imagery (Gentili et al., 2006). Kinesthetic imagery training improved performance to a degree comparable, albeit inferior, to physical training, as reflected in lower movement duration and increased peak acceleration, whereas performance was unchanged in both control groups. Interestingly, both the kinesthetic imagery and the physical training groups showed transfer of the acquired skill to the 
untrained, non-preferred hand at post-test.

In general, these findings support the view that optimal performance is underpinned by strengthened links along neural pathways dedicated to motor behavior, which thus can be trained by generating detailed simulations of motor actions. The evidence for transfer of skills to the untrained hand is interpreted by Gentili and colleagues (2006) as reflecting the neurocognitive mechanism of motor prediction, which enables the motor system to learn the relationship among forces in a given workspace, and to apply this knowledge to new states. Thus, cognitive enhancement from motor simulation is an important hallmark of cognition. However, data from the physical and mental training groups do suggest that overtly executing motor behavior might ultimately be more beneficial to performance. A convincing hypothesis acknowledged by the authors, also consistent with the predictive-coding approach (e.g., Clark, 2016), is that the relative disadvantage of kinesthetic imagery training, compared to physical activity, is ascribed to the lack of sensory feedback from the periphery which would allow for correction of the motor action. This interpretation is further supported by the finding that performance for the untrained hand, for which no sensory feedback was ever provided to the participants in either of the conditions, was enhanced to a similar degree in both kinesthetic imagery and physical training groups.

In a neuroimaging study of kinesthetic imagery, Guillot and colleagues (2007) assessed subjective levels of kinesthetic imagery skills through a battery of wellestablished tests in which the final score included four parameters: (a) levels of skin conductance response, which measured autonomic nervous system scores; (b) the Motor Imagery Questionnaire, which assessed the vividness of motor simulations; (c) an autoestimation score, which indicated mean within-subject ratings; and (d) a mental 
chronometry score which measured time differences between performed and imagined actions, and was thus negatively correlated with kinesthetic imagery ability levels. During fMRI scanning, participants were instructed to either perform a previously learned finger-tapping sequence, or to imagine performing the sequence, or to just listen to sounds. Compared to good imagers, poor imagers had increased activation in bilateral striatal areas, as well as the cerebellum and posterior cingulate and orbitofrontal cortices, often associated with the early acquisition stages of sequential movement and memory encoding, respectively. Good imagers, in contrast, showed more focused activation patterns in superior and right inferior parietal lobules, lateral premotor cortex, and left cingulate and right inferior prefrontal cortices, consistent with previous research highlighting this network as crucial to performing skilled motor behavior. This result illustrates once more how motor simulation enhances cognition. However, actual performance was not affected by imagery abilities, suggesting that the observed differences in brain activation patterns can be safely attributed to the recruitment of separate neural substrates, rather than on motor outcomes (Guillot et al., 2007).

As already noted for manipulations of physical effort (see Figure 2), the duration of the effects on cognition elicited through this imagery approach is also limited to a relatively short timescale, since the data in the reviewed studies were obtained within minutes after kinesthetic imagery practice. Moreover, the immediacy of such effects has also been established in the opposite direction, i.e., it has been demonstrated that a 10 minute session of physical exercise can boost cortical activation in premotor and motor areas related to imagery both during learning in healthy participants and during rehabilitation for brain damaged patients (Wriessnegger, Steyrl, Koschutnig, \& MüllerPutz, 2014). However, combining imagery and physical practice was found to 
contribute more to enhanced performance than both practices in isolation (Kalicinski \& Lobinger, 2013), and can thus be hypothesized to constitute one of the factors relevant to achieving long-lasting motor expertise.

In conclusion, research in mental imagery builds upon and provides support for the usefulness of integrating the basic mechanisms posited by embodied cognition accounts into training strategies tailored for specific goals.

\subsection{Motor training}

Another approach to studying the interdepencies and specific mechanisms bridging physical exercise and cognition is the integration of physical and cognitive demands into motor training programs (for overviews see Hillman, Erickson \& Kramer, 2008; Pesce \& Ben-Soussan, 2016). For instance, Moreau et al. (2015) developed a "complex motor training" paradigm by integrating motor, perceptual and cognitive problems into a movement-based framework "loosely based on freestyle wrestling" ( $p$. 46). In this newly designed physical activity, appropriate motor behavior depends on the outcomes of working memory (WM) and problem-solving tasks, which in turn are based on the previous learning of motor sequences, or on the position of the subject's body relative to other players. To test the validity of this training program, 67 participants were assigned to practice either complex motor training, a computerized WM-training, or aerobic exercise, for three hours per week over eight weeks. Weekly recordings of physiological measures included resting heart rate, blood oxygen and blood pressure levels; cognitive performance was assessed through a battery of WM and spatial-ability tasks before and after training. After eight weeks, all groups had improved on the scale targeted in each intervention. Aerobic exercise and motor training lowered blood pressure and resting heart rate, parameters typically associated with 
general health and longevity. However, aerobic exercise had no effect on cognitive measures, possibly attributable to the duration of the intervention, which was shorter than the more common 6- or 12-month interventions aimed at investigating cognitive benefits from general physical exercise (Moreau, 2015). Likewise, WM training did not improve any of the measured biomarkers. Notably, in addition to enhanced physical health, the target group showed higher improvements of cognitive outcomes than computerized WM training; specifically, complex motor training enhanced outcomes in both spatial ability and WM, whereas WM training exclusively improved WM performance. These findings seem to emphasize that cognitive training paradigms enhance cognition in a rather specific and somewhat trivial way - i.e., that training one skill typically results in better performance in that skill. More interestingly, these results from Moreau et al. (2015) provide support for the view that integrating physical and cognitive challenges into one activity not only contributes to optimizing brain function in terms of allocation of physiological resources, but presumably offers further advantages related to increased speed and accuracy of sensorimotor integration, which in turn produces benefits on both motor and cognitive performance.

In a separate line of research, Nei Gong training, a Chinese mind-body exercise, was tested as a tool to improve memory deficits typically found in children with autism spectrum disorder (ASD; Chan et al., 2015). Memory impairments in autistic patients are typically attributed to executive dysfunction, resulting in the inability to use strategies to integrate and retain information across contexts, which has been found to rely on the functional coupling of prefrontal and posterior parietal cortices (Nyberg et al., 2003). During Nei Gong training, participants train to maintain a relaxed, attentive mindset, integrated with simple, specific bodily movements. The rationale for this kind 
of intervention was based on previous research suggesting that memory performance in patients with severe memory impairments due to ASD was only slightly below the healthy average after just one month of Nei Gong training (Chan et al., 2015). This training paradigm has been suggested to foster awareness and self-control, and preliminary data suggested that Nei Gong training may underpin enhanced connectivity between prefrontal, temporal and limbic areas in ASD patients, which in turn play a central role in mediating memory, attention allocation and emotional processes on an intermediate time-scale (see Figure 2).

In Chan et al.'s (2015) study, 66 children with ASD were tested for baseline intellectual functioning and their baseline EEG was recorded during a visual memory task. Then, participants were randomized into a Nei Gong training group, an active control group assigned to progressive muscle relaxation, and a passive control group. The muscle relaxation group represents an important condition controlling for the positive effects of decreased stress on cognition. After four weeks, the childrens' visual memory was again assessed. Twelve images depicting items belonging to four distinct semantic categories (e.g., food, clothing) were presented either randomized or organized according to their category. After three minutes, another set of drawings was shown and participants had to recognize previously learned items among an equal number of distractors. The dependent variables included scalp EEG activity during encoding and total recall score, as well as semantic clustering and visual scanning scores, which are indicative of memory retrieval strategies. Semantic clustering is calculated as number of consecutively identified target items, which belong to the same semantic category, whereas in the visual scanning score the items consecutively retrieved belonged to similar visual locations during encoding. Participants in the Nei Gong training group 
outperformed both control groups in memory recall and retrieval strategies measures as a result of this embodied training. The authors concluded that Nei Gong training aided the spontaneous development of novel mnemonic strategies, such as grouping semantically-related content, as reflected in improved memory outcomes. EEG data showed that these behavioral changes were underpinned by higher theta coherence, as indicated by higher coherence values at $85 \%$ of electrodes in the target group, compared to $15 \%$ in the active and no changes in the inactive controls. Finally, increased current density in the Nei Gong training group indicated enhanced functional connectivity in the network between prefrontal, parietal and temporal cortices, and was interpreted by the authors as electrophysiological correlate of effective semantic categorization processes (Chan et al., 2015).

In summary, this approach has shown the effectiveness of motor training paradigms for enhancing cognitive performance on a timescale spanning from weeks to months, thereby supporting the embodied cognition perspective for the role of action in both on-line and off-line cognition (see Figure 2). Furthermore, it seems that for a structured training program to be effective, tailored interventions integrating cognitive and motor components should be informed by individual ability levels, both in healthy participants and in clinical populations, along with particular goals and areas of expertise targeted in each case, so as to adapt difficulty levels and render training approachable but challenging at all times (Moreau, 2015).

\subsection{Motor expertise}

In an early account of expert performance, deliberate practice was proposed by Ericsson and colleagues (1993) to constitute a crucial factor leading to excellence in a wide range of skills merging cognitive and motor challenges. As opposed to indirect and 
social learning, deliberate practice aims at achieving expert performance by engaging in activities specifically designed to maximize domain-specific improvements. In this framework, the linear relationship between improvement and amount of deliberate practice is considered a necessary and sufficient condition to achieving optimal performance. Famously, the authors claimed that deliberate practice should be sustained for a minimum of 10 years, or 10,000 hours, to attain expert performance, and further argued that protracted deliberate practice activities substantially contribute to long-term effects on performance by driving gene expression during development (e.g., in music and artistic practice; strategy and teamwork in sports, etc.; Ericsson, Nandagopal, \& Roring, 2009). Although it is unquestionable that practice is beneficial to performance as opposed to not practicing, the "monotonic" relationship between amount of practice and performance has been criticized as an untenable basic assumption, which disregards not only genetics, but also motivational and emotional factors (e.g., North, 2012).

As illustrated in this section, embodied cognition expands this field of research by providing conceptual parameters which are proven to be relevant in the study of expert performance (e.g., Raab, 2017), as well as revealing a range of factors influencing performance outcomes which are not accounted for by Ericsson and collaborators (1993; 2009), for instance decision-making strategies (Raab \& Laborde, 2011) and attention to bodily signals (Christensen, Gaigg, \& Calvo-Merino, 2018). We report these two studies next to illustrate the value of an embodied cognition approach to cognitive enhancement.

In embodied cognition research, motor expertise has been investigated as a potential source of domain-specific individual differences in the modulation of the MNS. In particular, expertise in domains which demand multisensory integration, such 
as dancing and playing music, has been related to better interoceptive accuracy, as well as modulating the level of kinesthetic imagery elicited by domain-relevant stimuli (Christensen et al., 2018). Specifically, striatal brain areas typically show decreased activation as a function of expertise, suggesting that motor representations are flexibly coded in sensorimotor regions over time and that implicitly executed (i.e., simulated) motor behavior requires fewer neural resources, while modulating alpha wave (de)synchronization (Di Nota et al., 2017). In addition to a positive correlation between interoceptive accuracy, interoceptive awareness, and years of dance practice, Christensen and collaborators (2018) also found evidence for a moderately strong association between accuracy and general art experience, although this correlation was limited to the dancers' group. This link was interpreted by the authors as suggestive of a role of attention in visual art training. Further, the results provide evidence for the effects of "any training involving both (a) elicitation of - and attention to - bodily signals $[\ldots]$, as well as (b) the use of these signals for the expression of states and emotions" (p. 9), in line with embodied cognition and converging with conclusions from other research reported in this review. This interpretation is supported by embodiment signatures found in art appreciation (Topolinski, 2010; Leder, Bär, \& Topolinski, 2012). In a study with handball players, Raab and Laborde (2011) analysed outcomes of intuitive and deliberate decision making as a function of level of expertise and gender. Expert, near-expert and non-expert players were categorized according to their league level - notably, non-experts were not complete beginners, since some degree of intuitive decision making was required to complete the task. Control variables included age, years of training and tactical knowledge. During the experimental procedure, participants were shown video clips of handball game actions and asked to generate, for 
specific frozen frames, (a) the first option for the player in ball possession; (b) further appropriate options; (c) which of the named options they thought would result in the best action course. Speed and accuracy were equally stressed in the instructions, so as to encourage experienced participants to respond using automatic information processing as well as affect-laden information - e.g., by relying on perceived somatic markers (Raab \& Laborde, 2011). Based on previous research, intuitive decision making was expected to produce faster and better choices, to enable participants to generate a lower number of higher-quality options, and thus to be correlated with level of expertise.

As expected, intuitive decision making led to faster and more correct responses than preference for deliberate decision and was robustly correlated with the level of expertise. Moreover, female players showed a slightly higher tendency to rely on intuitive decision making than male players. These results provide support for a heuristics model based on the idea that intuitive and deliberate decision-making strategies result in different patterns of choice generation. Corroborating the crucial role of intuitive decision-making in optimal sport performance, increasing levels of expertise have been shown to produce a "less-is-more" effect on performance, by which "the serial position of a generated option [is] inversely related to its quality, and an increase in generated options [reduces] the quality of the final choice" (Johnson \& Raab, 2003, p. 226).

A theoretical account for the interaction of cognitive and motor components of decision making has been put forward by Raab (2017), extending concepts from embodied cognition to account for motor heuristics in complex sport behavior. In a holistic perspective, motor heuristics refer to "fast-and-frugal" search, decision and execution rules which allow athletes to quickly choose between different options, in 
much the same way that simple heuristics allow selection of the most correct outcome for a cognitive task with limited time and cues. Whereas motor heuristics inform motor execution, the concept of embodied choices is introduced as the mechanism by which choices are generated, namely by means of the integration of prior sensorimotor experiences with information about the current position and state of the body, which are also identified as relevant cues to determining decision making and performance. This embodied cognition framework offers the advantage of capturing complex behavior and the interactions of parallel processes, while stressing the simplicity of heuristics models in accounting for the role of several factors involved in decision-making. Although its applicability has not yet been tested empirically to inform training and coaching methods in sports, this framework offers a holistic perspective for implementing domain-specific, tailored heuristics training and diagnostic tools for athletes (Raab, 2017). Such a simple heuristic approach is in contrast to approaches that correlate wellestablished cognitive processes (e.g., executive funtions and their subconstructs) to sport performance (e.g., in soccer performance indicators) - a practice that does not allow to understand the origin of effects and whether expert-novice differences are due to training or selection (e.g., Jacobsen \& Matthaeus, 2014; Verburgh, Scherder, van Lange, \& Oosterlaan, 2014; Vestberg, Gustafson, Maurex, Ingvar, \& Petrovic, 2012).

\section{Embodied learning}

Insights from embodied cognition have informed the development of novel paradigms and strategies to enhance learning outcomes in adults, children and clinical populations, particularly in the domains of Science-Technology-EngineeringMathematics (STEM) education, language and memory (Weisberg \& Newcombe, 2017). Similar to research reviewed above, education studies have focused particularly 
on incorporating perceptuo-motor activities and cognitive challenges into learning programs suited for specific learning goals and populations. Embodied parameters have been integrated to different extents into experimental interventions where the focus varies from highlighting the relevance of instructed and/or spontaneous gesturing to successful learning, to promoting embodied interaction with physical systems relevant to the subject, to immersing learners into augmented reality simulations of learning environments. We will review these three domains in turn (see Figure 3 for an overview).

\section{Insert Figure 3 about here}

\subsection{Gesturing}

Qualitative research has investigated the general observation that learners are often found to spontaneously use their bodies as cognitive tools to learn and illustrate old and novel concepts (e.g., Davidsen \& Ryberg, 2017; Eskildsen \& Wagner, 2015). Davidsen and Ryberg (2017) noted that 9-year-olds, whose interactions they analysed through video materials, used bodily-material resources to accomplish different communicative and learning goals about the mathematical concept of scale. Bodilymaterial resources include gestures, touch, body positions and movements, and have been found to play a crucial role not only for communicative and illustrative purposes, but importantly also as cognitive auxiliary tools, for instance in finger-counting (Fischer \& Brugger, 2011) and in concretizing concepts into flexible, short-lived semiotic resources, such as counting by nodding or moving the hands while reasoning (Carlson, 
Avraamides, Cary, \& Strasberg, 2007). Bodily-material resources are also thought to play a role in shepherding and instructing peers, in a way that often complements language in a multimodal utterance (Davidsen \& Ryberg, 2017).

Similarly, Eskildsen and Wagner (2015) used conversation analysis to investigate the coupling of gestures and specific language expressions during second language (L2) learning. Their analysis of the development of specific gestures along with linguistic constructions in L2 learners of English indicated that these are functional to L2 learning as a communication strategy when difficulties arise. Eskildsen and Wagner (2015) considered two linguistic items, under and across, and their relationship with specific gestures in different learning occasions, and associated with different linguistic constructions including these items. First, specific gestures associated with either of the items were deployed to display understanding during learning, and used concomitantly with the same constructions in later relevant situations, particularly when difficulties in speech production were encountered. Moreover, the analysis of gestures produced after learning of the specific items highlighted that these seem to emphasize subtle semantic nuances which are not explicitly differentiated in linguistic constructions sharing the same item (e.g., across and across from; Eskildsen \& Wagner, 2015).

In language studies, the relevance of embodied parameters to knowledge retention has been demonstrated by inventing techniques to aid vocabulary development. In a report by Hald, de Nooijder, van Gog, and Bekkering (2016), results from studies of vocabulary knowledge are reviewed and commonalities among successful training programs are highlighted. In particular, results from 41 of 44 reported vocabulary training studies seem to share three main aspects: In line with 
embodied cognition, as well as with recommendations already made by the pioneering pedagogue Maria Montessori (Lillard, 2005), the effectiveness of vocabulary training interventions was mainly determined by (a) sensory richness of the learned words; (b) establishment of links between meaning and previous (motor) experience; and (c) the extent to which movement was integrated into vocabulary training (Hald et al., 2016). Moreover, whereas simple presentation of multimedia information produced no beneficial effects, interactive learning material aided word learning, again suggesting that the advantages of incorporating sensorimotor richness into learning programs go beyond the effects of simple multimodal repetition (Hald et al., 2016). The sensorimotor system, the authors conclude, seems to consistently underlie the strengthening of congruent ties between learning content and individual motor experience, which are beneficial to cognitive processing and knowledge retention.

One well established signature of cognitive embodiment is the enactment effect on memory which refers to improved retrieval when to-be-remembered items were encoded through motor actions (e.g., Jaroslawska, Gathercole, Allen, \& Holmes, 2016; Sidhu \& Pexman, 2016). Hainselin and colleagues (2017) recently investigated the effect of enactment on memory in French elementary school children by comparing four different conditions in 6- and 10-year-olds. 35 children from both groups encoded 24 poorly integrated action-phrases and were assigned to either reading out loud, listening to, watching or performing the actions referred to in the sentences. The linguistic stimuli were formulated in such a way that children would have to couple an action and an object in a novel combination, which is not commonly encountered, thus excluding the confound of children's prior experience with everyday actions. After encoding, the successful retrieval of the stimulus actions was assessed in successive free and cued 
recall tasks; actions which were not retrieved correctly, or not at all, were presented in the learned context together with two distractors in a forced-recognition task, and children were directly asked which of the actions had been encoded in that context. The results showed enhanced memory performance for both performing and observing actions compared to the reading and listening conditions, particularly in free recall scores, and regardless of age group. Moreover, no difference in cognitive performance between 6- and 10-year-olds was detected when encoding included action performance, but the cognitive gap was again evident in the observation condition. In general, participants who enacted the movements outperformed all other groups with an $80 \%$ success rate (Hainselin, Picard, Manolli, Vanderkore-Candas, \& Bourdin, 2017).

Empirical evidence from education research has also suggested that spontaneous gesturing might correlate with better encoding of new problems, knowledge retrieval and memory consolidation. However, the extent to which gestures passively reflect or functionally aid ongoing cognitive processes had not been explored systematically until relatively recently. In a developmental study, Cook and colleagues (2008) hypothesized that, if gestures merely reflect cognitive processing without altering it, knowledge acquisition and retention should not be influenced by simultaneous gesturing. If, on the contrary, gestures play a functional role in cognition, they should have a measurable effect on learning. To examine these predictions, 84 third- and fourth-grade children were first tested to verify that none of them was able to solve a certain mathematical problem. Then, the experimenters gave children explicit instructions about how to solve the problem. The participants were encouraged to illustrate the strategies used to find a solution either with gestures only, combining gestures and speech, or with speech only. After the learning session, progress made by children in the gesturing groups was 
compared to that of children instructed to explain the same strategy in words. Moreover, a follow-up assessment at 4 weeks included a similar test administered during school hours by the child's classroom teacher, and aimed to measure retention and generalization to new contexts. Children in all three groups solved a comparable number of problems during instruction and immediate post-test. However, whereas verbal instruction had helped children perform the task immediately after training, it only showed a weak correlation with learning retention at the follow-up assessment. In contrast, a strong effect of gesturing, with or without accompanying speech, was found on performance at the 4-week follow-up. Specifically, performance outcomes of the gesturing groups at immediate post-test were predictive of outcomes measured one month later. Children who gestured maintained $85 \%$ of their learning gains, whereas children who just repeated the teacher's instructions only retained 33\%. Interestingly, gesturing did not seem to have an effect on immediate learning, possibly because verbal instructions also effectively helped children to gain the knowledge they needed for the task. However, only children who used gestures actually internalized the concept and were able to apply it to new situations.

In a study illustrating the embodied approach to memory enhancement, van Dam and coworkers (2013) considered the evidence that perceptual and action-related information is coded in brain systems underlying specific modalities of sensorimotor processing. Thus, the researchers tested the hypothesis that memory performance is enhanced by engaging the sensorimotor system in a way compatible with the learning content. In this study, 21 university students learned a list of nouns referring to manipulable objects: half of them implied a twisting movement, while the other half were manipulable with a pressing movement; a list of neutral nouns served to establish 
baseline memory outcomes. After encoding, participants underwent a retention phase, in which a distractor task included a further manipulation by either giving twisting or pressing responses. Motor responses were thus either congruent or incongruent with the learned items. Finally, a delayed recognition memory task assessed mean hit and falsealarm rates for congruent and incongruent trials. The results revealed a main effect of congruency, confirming the hypothesis that performing gestures compatible with learning content, even in different time windows, enhanced memory performance. In two follow-up experiments, the authors demonstrated that congruent action responses carried out during the retention phase also enhance early recognition of manipulable objects in a picture fragmentation test, a measure shown to be insensitive to semantic factors (van Dam et al., 2013). This finding enabled the authors to conclude that memory enhancement is unlikely to be due to semantic priming induced by motor responses in the retention phase. Rather, the observed effects of enhanced memory consolidation seem to be directly driven by the congruent motor manipulations.

Recently, further research has aimed at detecting effects of congruency and relative timing of verbal and gestural instructions, as well as determining the extent to which long-term learning relies on each of both explicit and implicit modalities. For instance, Brooks and Goldin-Meadow (2016) tested the prediction that, if gestures are presented prior to explicit instructions, they should influence children's learning. Specifically, compatible gesturing is expected to positively affect the understanding of mathematical problems, whereas incompatible gesturing should have a negative effect. Notably, the experimental design isolated gesture production from explicit instruction, and presented mathematical problems along with compatible or incompatible gestures prior to verbal instructions. At this point, the number of correct answers and number of 
explanations of correct strategies to solve the problem were collected. After children received explicit instructions on how to solve the problems, their performance was assessed in a post-training test presenting the same kind of task. Results showed no changes in children's knowledge after gesturing and before explicit instructions were provided, whereas significant differences in performance of the post-instruction task indicated that compatible gesturing had positively influenced understanding and learning of novel strategies. Interestingly, the qualitative analysis of results showed that children generated correct explanations for strategies that they were not able to produce in words. The authors concluded that gesturing might have a "sleeper" effect on children's math learning, suggesting that compatible gesturing did not influence the children's representations of problems, but rather helped them by emphasizing the salient aspects of the task, whereas incompatible gestures might have prevented children from encoding relevant elements in the verbal instructions.

These findings also seem to support the view that gesturing plays a role even when it does not occur concomitantly with verbal instructions, with important practical implications for the development of interfaces for cognitive training apps on touchscreen devices (Brooks \& Goldin-Meadow, 2016). However, Congdon et al. (2017) showed that instructions containing simultaneous speech and gestures aided $3^{\text {rd }}$ graders in learning and generalizing knowledge, compared to when verbal and gestural instructions were given successively. In the experimental set-up, all children were given the same two strategies to solve a problem. One group received instructions for both strategies through speech; another received successive instructions, first through speech for one strategy and then through gestures for the other; and a third group was given instructions simultaneously in both modalities. No difference was found between groups 
immediately after the training. However, children who were instructed simultaneously with speech and gestures showed the best outcomes in generalization of strategies to problems presented both at 24-hour and 4-week post-training assessments. In contrast, learning outcomes at 4 weeks did not differ between groups presented with successive instructions. Congdon and colleagues (2017) interpreted the apparent discrepancy of their results with previous findings (e.g., Cook, Mitchell, \& Goldin-Meadow, 2008) as a consequence of the fact that, in their study, only the instructors were producing the gestures, not the learners. Thus, they hypothesized that gestures might need a tight coupling with speech when observed in others, but this appears to be less critical when gestures are actively produced by learners.

In sum, the reported studies provide strong support for the validity of paradigms integrating gestures with explicit learning instructions in interactive environments, as observed in spontaneous behavior in qualitative studies and predicted by embodied accounts of cognition. Several mechanisms have been proposed to underlie the observed cognitive advantages offered by gesturing. First, gestures might represent a way of offloading some of the cognitive processing onto bodily movements. Since these are relatively easy to prompt, expressing information with both speech and gestures may require less effort than with speech alone, as reported e.g. in Eskildsen and Wagner (2015). Second, gesturing might directly aid memory by engaging motor-related brain structures in the encoding process, thus strengthening memory traces more than verbal instruction alone (Hainselin et al., 2017). Third, by engaging with the environment, learners can link learned strategies and content to relevant features of the educational setting (Cook et al., 2008). 


\subsection{Embodied interaction}

Recently, several paradigms have been developed based on the interaction of learners with actual physical systems which highlight relevant aspects of the learning content. These design decisions aim to increase the specific sensory-motor stimulation that supports learning. Particularly within STEM education and memory research, the embodied framework has been validated as a means to improve learning, reasoning and memory outcomes in both high-school (Gregorcic, Planisic, \& Etkina, 2017) and college students (Kontra, Lyons, Fischer, \& Beilock, 2015). We discuss these latter two studies here to further illustrate the close connection between embodied cognition and cognitive enhancement.

Gregorcic and collaborators (2017) examined spontaneous bodily movements produced by a group of high school students towards an interactive whiteboard equipped with content about the orbital motion of planets. Multimodal discourse analysis was used to observe which meaning-making strategies were employed by the student group in the educational setting, and how closely these modalities resembled actual scientific practices. A particular focus of the analysis was the integration of language, gesture and interaction with education tools when engaging in scientific inquiry practices. The results indicated that students rely heavily on means other than speech to understand and convey new information, with simple gestures as well as gestures referring to the content displayed by the learning tool. Moreover, in line with observations by Brooks and Goldin-Meadow (2016) and by Eskildsen and Wagner (2015), it was evident that gestures did not simply accompany speech, but rather integrated and expanded it in a non-redundant way. In addition, gestures enabled students to incorporate environmental features in their reasoning process by closely 
juxtaposing self- and tool-referred movements. Finally, the interactive whiteboard not only allowed physical engagement with the workspace parameters, but also encouraged students to directly test experimental hypotheses in a way akin to scientific investigation.

Kontra and colleagues (2015) showed that physically interacting with relevant properties of learned concepts also enhanced understanding and reasoning skills in college students. In this study, the authors investigated the effects of directly experiencing the consequences of angular momentum vectors, which was expected to enrich the students' concepts about the learning materials with additional sensori-motor information. In the target group, students manipulated the axle of a two-wheel device, whose spinning and tilting motion varied as a function of the wheels' size, relative position and spin direction. The consequences of the participants' interaction with the device were visible to an observation group, which served as control. For students in the target group, significantly greater levels of understanding were reported than for the observation group, as measured by a quiz task score. A randomized field experiment with a different sample assessed the students' performance on the same concepts several days after the manipulation and after explicit instructions had been provided. The action group was again found to outperform the observation group, especially for the questions in the quiz which were focused on reasoning with vectors. These findings seem to fit embodied predictions about the beneficial role of physical experience on the ability to comprehend and reason about STEM subjects.

Furthermore, Kontra and coworkers (2015) used fMRI to detect the neural underpinnings of enhanced learning due to physical interaction. For the action group, increased activation was found at post-test in right dorsal premotor, primary motor and 
somatosensory cortices, the superior parietal lobule, supplementary motor area and cerebellum compared to the observation group (Kontra et al., 2015). That these patterns of activation were stronger in the left hemisphere is likely due to the fact that all participants were right-handed, although a bilateral component was detected in M1 and S1 activations with a less conservative threshold. The level of activation in this network of brain regions was found to be predictive of quiz score accuracy and to be modulated as a function of training group, thus leading the authors to conclude that these findings establish a causal link between sensorimotor experience and enhanced learning outcomes about dynamic physical concepts.

Embodied interaction with learning material has proven effective also in clinical populations. In a recent study by Trevisan, Sedeno, Birba, Ibanez and Garcia (2017), 20 dyslexic children underwent an intervention consisting of 90-minute supervised sessions of whole-body videogame-based training on a Nintendo Wii console, spread out over the course of nine days. A separate sample of 10 children served as control group, for which the same procedure was carried out, but the videogame only required minimally embodied keystroke responses on a joypad. The mean age was 9.8 years and the groups were matched for handedness, years of education and gender. Moreover, no speech therapy or videogame session was allowed to either group outside the laboratory for the duration of the study. Before and after the treatment, two brief stories were read out to the participants narrating a day in the life of different characters. The two experimental conditions compared an abstract-text condition, in which $80 \%$ of the verbs in the story implied no physical action, to an action-text condition, which included $70 \%$ actionrelated verbs. Thus, participants were shown one abstract and one action-text at pre-test, and both remaining texts were presented at the post-test assessment. The texts were 
thoroughly matched by the researchers on several linguistic aspects, following semantic, syntactic and distributional criteria, as well as controlling for length, frequency, familiarity and imageability of both single words and whole sentences. At both assessments, the texts were followed by a phonological short-term memory task and a 16-item multiple choice questionnaire about the content of the stories. Half of the questions regarded information conveyed by action verbs, while for the other half, the information was carried by adverbial or prepositional phrases. For the target group, the results showed that comprehension of information conveyed by action verbs was significantly enhanced after the videogame-based bodily training compared to information conveyed by adverbial and prepositional phrases. These results are consistent with previous findings showing that attention to visuospatial patterns triggers activation in the magnocellular dorsal pathway, typically engaged in motor action. This effect remained significant after considering the observed enhancement of phonological short-term memory performance as a covariate in the analyses. Thus, engaging the sensorimotor system and action imagery seem to have a generalized, coarse-grained impact on semantic processes, particularly on the appraisal and comprehension of action-related language, along with the finer-grained recruitment of relevant brain areas reported in previous research (e.g., Fischer \& Zwaan, 2008; Pulvermüller, 2013). However, future research would need to further investigate the extent to which videogame-based bodily training can be used to enhance also language production in dyslexic patients and individuals with action-specific linguistic deficits, to create diagnostic tools for patients suffering from motor disorders (e.g., Parkinson's disease) and to assist L2 learning in healthy participants.

In summary, embodied interactions with learning materials during didactic 
interventions have demonstrated their effectiveness at enhancing learning outcomes across the developmental time line. The usefulness of insights from embodied cognition in didactic settings is supported by the evidence for an effect of relevant movements on cognitive performance (e.g., Kontra et al., 2015) and of generalized movement for therapy outcomes in patients with language disorders (e.g., Trevisan et al., 2017). The evidence reviewed supports the role of physical interaction and interactive tools not only in learning the targeted information, but also in developing a scientific mindset, which is of primary relevance to STEM research and education in general. In future research, similar therapeutic and learning paradigms could be applied to other populations to aid rehabilitation from stroke and therapy for motor and linguistic disorders, as well as informing standard didactic programs and L2 learning strategies.

\subsection{Augmented reality}

In a recent line of research, more immersive technologies have been deployed in order to implement insights from embodied cognition into learning paradigms. The effectiveness of augmented reality (AR) has been tested by Johnson-Glenberg and collaborators (2016) in a study focusing on the effects of different levels of embodiment on learning gains in college students. The rationale behind this investigation builds on the hypothesis that platforms integrating embodied interaction and real-time feedback might facilitate knowledge acquisition and retention. First, 109 participants received explicit instructions about centripetal force. During the educational intervention, participants were randomized to one of three learning platforms: a desktop animation, an interactive whiteboard or SMALLab ${ }^{\odot}$, an immersive AR platform which allows the user to control virtual simulations with whole-body activity. In addition, the level of embodiment was manipulated for all platforms in such a way that low embodiment 
conditions included relatively narrow movements and required adjustments of the relevant variables through button-pressing. In contrast, high embodiment conditions required wide, whole-body movements and active participation in carrying out the virtual simulation. Specifically, the high embodiment level was operationalized in SMALLab $^{\odot}$ through responses requiring whole-body activity and locomotion, while the whiteboard required wide whole-body movements, and the desktop animation condition required continuous interaction with the relevant variables until task completion. Participants were then tested immediately after the experimental manipulation and one week later, to separately assess learning gains and knowledge retention. Contrary to the authors' expectation, the results showed no effect of learning platform manipulation when the data were collapsed for both embodiment levels. Embodiment level, however, was found to be predictive of knowledge retention, particularly in generative tasks, although performance at the immediate post-intervention assessment did not differ between groups.

The unexpected benefits for immediate learning for all groups were interpreted as an effect of using state-of-the-art technology in the interventions, which even in the low embodiment condition might have led to greater embodiment, sense of agency and student engagement than education devices commonly present in schools or on the market. In other words, all conditions offered the opportunity of engaging with the relevant aspects of centripetal force more than in regular classroom settings. Moreover, the authors declare that it "would have felt somewhat unethical" (p. 16) to consciously allow students to leave the intervention with incorrect mental models, and corrective guidance was provided when students were not able to produce the correct response during the intervention. This could also have contributed to the indistinguishable levels 
of performance at immediate post-test. In summary, these findings bolster the view that embodied parameters can be effectively applied to enhance long-term learning gains, and specifically in the design of both learning materials, which should promote sensorimotor engagement and multimodal integration, and interactive tools for educational purposes, in particular within STEM subjects (Johnson-Glenberg, Megowan-Romanowicz, Birchfield, \& Savio-Ramos, 2016; Weisberg \& Newcombe, 2017).

Parallel research has emphasized the role of interactive AR platforms as contributors to potential changes in dispositional affect towards learning contents and science at large, as well as self-efficacy. Indeed, increased motivation and engagement are typically observed in school subjects that are of particular individual interest for students, as well as being predictive of learning strategies and academic success (Lindgren, Tscholl, Wang, \& Johnson, 2016). In this study, the impact of the degree of embodied interaction was investigated both on learning gains and on feelings about the simulation and about their own abilities to relate to science. In an experimental set-up similar to Johnson-Glenberg et al. (2016), Lindgren et al. (2016) presented middleschool students with either a desktop animation or an AR simulation of planetary astronomy. In addition to cognitive measures, the participants were asked a set of question to assess the degree of presence they felt to the content, as well as their feelings towards the simulation, and the degree to which they thought that the simulation helped them to learn. These questions were drawn from three subcategories of attitudes towards science: enjoyment of science, value of science in society and selfconcept. The results confirmed the authors' expectations regarding enhanced learning outcomes for the AR simulation group compared to desktop animation. In the 
discussion, they argued that not physical interaction alone, but rather the coupling of everyday movements with challenging science concepts within the simulation, allows learners to merge sensorimotor perceptions with representations of the relevant aspects of learning materials. Familiarity with the movements involved in the interaction decreases the perceived distance to the learned concepts and to scientific knowledge in general, which is often associated in the classroom with rote learning of highly abstract symbol systems. The authors conclude that embodiment might foster higher feelings of presence, engagement and self-efficacy, and that these aspects need to inform learning paradigms to target both students' knowledge development and affective dispositions (Lindgren et al., 2016).

Taken together, this evidence supports the validity of embodied manipulation at enhancing learning outcomes by fostering bodily interaction with the phenomena under investigation by means of platforms and interactive tools. Two findings, which were partially unexpected, seem to point to new directions for inquiry. First, the level of embodiment required by participants was found to contribute to knowledge retention, rather than the level of embodiment of the platform that was used (Glenberg-Johnson et al., 2016). This finding will have to be replicated and possibly further defined by controlling for confounding factors, such as reward and motivation, in future research. Second, embodiment of parameters of the relevant phenomena might in turn contribute positively to the learners' disposition towards and engagement with scientific subjects, as well as self-efficacy (e.g., Lindgren et al., 2016), which are important motivating factors to consider in designing learning programs.

\section{Conclusion}

This selective review of behavioral studies from the domains of physical 
exercise and embodied learning has pointed out the relevance of an embodied cognition perspective, according to which all our knowledge is multi-modal and tightly coupled with sensory and motor activation. This perspective has important implications for cognitive enhancement: First, it predicts the ubiquity of motor activation as a result of a simulation process that constitutes the core of all knowledge retrieval. Therefore, combining physical and cognitive tasks can lead to predictable interference or facilitation, depending on the specific instructions implemented. Secondly, it predicts whether the transfer of training effects from one domain to the other will be easy or hard, depending on the modality combinations implemented in a given dual-task (cf. Schaeffner, Koch, \& Phillipp, 2018). Third, the time-course of learning can be mapped out as a progression from short-term situated aspects of cognition, such as the momentary configuration of one's mental set, to the long-term cognitive enhancement effects intended by sensori-motor trainings.

Further support for embodied views on the enhanced mind and its health comes from clinical studies. For instance, Michalak, Troje, and Heidenreich (2010) showed how mindfulness-based cognitive therapy for depressed patients changes their clinical prototypical gait patterns. At the same time, analysing movement patterns may in the future allow to predict relapse of formerly depressed patients, as Michalak and colleagues showed that gait patterns of formerly depressed individuals at high risk of relapse are similar to those typically associated with depressive symptomatology. These and other examples (Michalak, Burg, \& Heidenreich, 2012; Cardona, 2017) illustrate the potential of embodied cognition to complement cognitive enhancement approaches by drawing attention to the close interplay between body and mind along multiple time scales. 
Embodied cognition also provides the theoretical framing desired by some proponents of cognitive enhancement who wish to replace "enhancement short-cuts" such as food supplements, drugs or gaming (e.g., Colzato, 2017, p. 323-4) with the development of theoretically driven training regimes inspired by an embodied understanding of cognition generally. This theoretical framework also permits consideration of individual differences as a result of idiosyncratic learning histories (cf. Casasanto, 2011) and thus prevents generalized enhancement claims that are supposed to fit all.

In summary, the evidence reviewed bolsters the importance of the body and of motor behavior for cognition and highlights new perspectives to enhancing cognitive capacities. Embodiment theory offers a novel, ecologically sound approach to cognitive enhancement, achieved through different sets of behaviors or training programs which are found to influence cognitive processing at varying timescales. The flexibility and non-invasiveness of the embodied approach to cognitive enhancement are relevant both for guiding developments in the design of new learning paradigms and tools, and for informing new therapeutic and rehabilitation strategies.

\section{Acknowledgments}

We thank Bernhard Hommel for helpful comments on an earlier version of this text. 


\section{References}

Alban, M. W., \& Kelley, C. M. (2013). Research report: Embodiment meets metamemory: Weight as a Cue for Metacognitive Judgments. Journal of Experimental Psychology: Learning Memory and Cognition, 39(5), 1628-1634. https://doi.org/10.1037/a0032420

Barsalou, L. W. (2008). Grounded cognition. Annual Review of Psychology, 59(1), 617645. https://doi.org/10.1146/annurev.psych.59.103006.093639

Bhalla, M., Proffitt, D. R., Bertamini, M., Bertenthal, B., Bunker, L., Creem, S., Gaesser, G., Kubovy, M., Yang, T. (1999). Visual-motor recalibration in geographical slant perception. Journal of Experimental Psychology: Human Perception and Performance, 25(4), 1076-1096.

Brooks, N., \& Goldin-Meadow, S. (2016). Moving to learn: How guiding the hands can set the stage for learning. Cognitive Science, 40(7), 1831-1849. https://doi.org/10.1111/cogs.12292.

Cardona, J.F. (2017). Embodied cognition: A challenging road for clinical neuropsychology. Front. Aging Neurosci. 9:388. doi: 10.3389/fnagi.2017.00388

Carlson, R. A., Avraamides, M. N., Cary, M., \& Strasberg, S. (2007). What do the hands externalize in simple arithmetic? Journal of Experimental Psychology: Learning, Memory, and Cognition, 33(4), 747-756.

Casasanto, D. (2011). Different bodies, different minds: The body specificity of language and thought. Current Directions in Psychological Science, 20, 378-383. https://doi.org/10.1177/0963721411422058

Chan, A. S., Han, Y. M. Y., Sze, S. L., \& Lau, E. M. (2015). Neuroenhancement of 
memory for children with autism by a mind-body exercise. Frontiers in Psychology, 6(DEC), 1-17. https://doi.org/10.3389/fpsyg.2015.01893

Christensen, J. F., Gaigg, S. B., \& Calvo-Merino,. B. (2018). I can feel my heartbeat: Dancers have increased interoceptive accuracy. Psychophysiology, e-bub ahead of print. doi: https://doi.org/10.1111/psyp.13008

Clark, A. (2016). Surfing uncertainty. New York: Oxford University Press. ISBN: 9780190217013

Coello, Y., \& Fischer, M. H. (2016). Foundations of embodied cognition - Volume 1. Perceptual and emotional embodiment. London, UK: Taylor \& Francis.

Colzato, L. S. (2017). Theory-driven approaches to cognitive enhancement. https://doi.org/10.1007/978-3-319-57505-6

Congdon, E. L., Novack, M. A., Brooks, N., Hemani-Lopez, N., O’Keefe, L., \& GoldinMeadow, S. (2017). Better together: Simultaneous presentation of speech and gesture in math instruction supports generalization and retention. Learning and Instruction, 50, 65-74. https://doi.org/10.1016/j.learninstruc.2017.03.005

Cook, S. W., Mitchell, Z., \& Goldin-Meadow, S. (2008). Gesturing makes learning last. Cognition, 106(2), 1047-1058. https://doi.org/10.1016/j.cognition.2007.04.010

Cremen, I. A., \& Carson, R. G. (2017). Have standard tests of cognitive function been misappropriated in the study of cognitive enhancement? Frontiers in Human Neuroscience, 11(May), 1-9. https://doi.org/10.3389/fnhum.2017.00276

Daamen, M., \& Raab, M. (2012). Psychological assessment in physical exercise. in H. Boecker, C. H. Hillmann, L. Scheef, \& H. K. Strüder (Eds.), Functional neuroimaging in exercise and sport sciences. (109-153). Berlin: Springer Science 
\& Business Media LLC.

Davidsen, J., \& Ryberg, T. (2017). "This is the size of one meter”: Children's bodily-material collaboration. International Journal of Computer-Supported Collaborative Learning, 12(1), 65-90. https://doi.org/10.1007/s11412-017-9248-8

Di Nota, P. M., Chartrand, J. M., Levkov, G. R., Montefusco-Siegmund, R., \& DeSouza, J. F. X. (2017). Experience-dependent modulation of alpha and beta during action observation and motor imagery. BMC Neuroscience, 18(1), 28. https://doi.org/10.1186/s12868-017-0349-0

Dietz, P. (2013). The influence of sports on cognitive task performance - a critical overview. In: Hildt, E. \& Franke, A. G. (Eds.) Cognitive enhancement: An interdisciplinary perspective. Trends in augmentation of human performance, 1. https://doi.org/10.1016/B978-0-12-417042-1.00010-3

Dresler, M. (2013). Behavioral neuroenhancement. In: Hildt, E. \& Franke, A. G. (Eds.) Cognitive enhancement: An interdisciplinary perspective. Trends in augmentation of human performance, 1. https://doi.org/10.1016/B978-0-12-417042-1.00010-3

Drid, P., Majstorović, N. \& Drapšin, M. (2010). The effects of different excercise workloads on visual perception skills in elite Serbian female judokas. Kinesiology 42, 201-207.

Dubljević, V., Venero, C., \& Knafo, S. (2015). What is cognitive enhancement? In Cognitive enhancement: Pharmacologic, environmental and genetic factors (pp. 19). Elsevier. North Holland.

Ericsson, K. A., Krampe, R. T., \& Tesch-Römer, C. (1993). The Role of Deliberate Practice in the Acquisition of Expert Performance. Psychological Review, 100(3), 
$363-406$.

Ericsson, K. A., Nandagopal, K. and Roring, R. W. (2009), Toward a science of exceptional achievement. Annals of the New York Academy of Sciences, 1172: 199-217. doi:10.1196/annals. 1393.001

Eskildsen, S. W., \& Wagner, J. (2015). Embodied L2 construction learning. Language Learning, 65(2), 268-297. https://doi.org/10.1111/lang.12106

Fernandes, H. L., Stevenson, I. H., Vilares, I., \& Kording, K. P. (2014). The generalization of prior uncertainty during reaching. Journal of Neuroscience, 34(34), 11470-11484. https://doi.org/10.1523/JNEUROSCI.3882-13.2014

Fischer, D., Messner, M., \& Pollatos, O. (2017). Improvement of interoceptive processes after an 8-week body scan intervention. Frontiers in Human Neuroscience, 11(September), 1-12. https://doi.org/10.3389/fnhum.2017.00452

Fischer, M. H. (2012). A hierarchical view of grounded, embodied and situated numerical cognition. Cognitive Processing, 13, S161-S164. DOI 10.1007/s10339012-0477-5.

Fischer, M. H., \& Brugger, P. (2011). When digits help digits: Spatial-numerical associations point to finger counting as prime example of embodied cognition. Frontiers in Psychology, 2(OCT), 1-7. https://doi.org/10.3389/fpsyg.2011.00260

Fischer, M. H., \& Coello, Y. (2016). Foundations of embodied cognition - Volume 2: Conceptual and interactive embodiment. London, UK: Taylor \& Francis.

Fischer, M. H., \& Shaki, S. (2014). Spatial associations in numerical cognition: From single digits to arithmetic. Quarterly Journal of Experimental Psychology, 67(8), 1461-1483. https://doi.org/10.1080/17470218.2014.927515 
Fischer, M. H., \& Shaki, S. (2018). Number concepts - abstract and embodied.

Philosophical Transactions of the Royal Society B, 373 (1752), 20170125. http://dx.doi.org/10.1098/rstb.2017.0125

Fischer, M. H., \& Zwaan, R. A. (2008). Embodied language: A review of the role of the motor system in language comprehension. The Quarterly Journal of Experimental Psychology, 61(March), 825-850. https://doi.org/10.1080/17470210701623605

Gentili, R., Papaxanthis, C., \& Pozzo, T. (2006). Improvement and generalization of arm motor performance through motor imagery practice. Neuroscience, 137(3), 761-772. https://doi.org/10.1016/j.neuroscience.2005.10.013

Gentsch, A., Weber, A., Synofzik, M., Vosgerau, G. \& Schütz-Bosbach, S. (2016). Towards a common framework of grounded action cognition. Relating motor control, perception and cognition. Cognition, 146, 81-89.

Glenberg, A. M., \& Gallese, V. (2011). Special issue: Viewpoint action-based language: A theory of language acquisition, comprehension, and production. Cortex, 48(7), 905-922. https://doi.org/10.1016/j.cortex.2011.04.010

Gómez-Pinilla. (2008). Brain foods: The effects of nutrients on brain function. Nature Reviews Neuroscience, 9(7), 568-78.

Gregorcic, B., Planinsic, G., \& Etkina, E. (2017). Doing science by waving hands: Talk, symbiotic gesture, and interaction with digital content as resources in student inquiry. Physical Review Physics Education Research, 13(2), 1-17. https://doi.org/10.1103/PhysRevPhysEducRes.13.020104

Guillot, A., Lebon, F., Rouffet, D., Champely, S., Doyon, J., \& Collet, C. (2007). Muscular responses during motor imagery as a function of muscle contraction 
types. International Journal of Psychophysiology, 66, 18-27. doi:

10.1016/j.ijpsycho.2007.05.009

Hainselin, M., Picard, L., Manolli, P., Vankerkore-Candas, S., \& Bourdin, B. (2017). Hey teacher, don't leave them kids alone: Action is better for memory than reading. Frontiers in Psychology, 8(MAR), 1-9. https://doi.org/10.3389/fpsyg.2017.00325

Hald, L. A., de Nooijer, J., van Gog, T., \& Bekkering, H. (2016). Optimizing Word Learning via Links to Perceptual and Motoric Experience. Educational Psychology Review, 28(3), 495-522. https://doi.org/10.1007/s10648-015-9334-2

Hillman, C. H., Erickson, K. I., \& Kramer, A. F. (2008). Be smart, exercise your heart: Exercise effects on brain and cognition. Nature Reviews Neuroscience, 9(1), 5865. DOI: $10.1038 / \mathrm{nrn} 2298$

Jacobson, J. \& Matthaeus, L. (2014). Athletics and executive functioning: How athletic participation and sport type correlate with cognitive performance. Psychology of Sport and Exercise, 15, 521-527.

Jaeggi, S. M., Buschkuehl, M., Jonides, J., \& Perrig, W. J. (2008). Improving fluid intelligence with training on working memory. Proc Natl Acad Sci USA, 105(19), 6829-6833. https://doi.org/10.1073/pnas.0801268105

Jaroslawska, A. J., Gathercole, S. E., Allen, R. J., \& Holmes, J. (2016). Following instructions from working memory: Why does action at encoding and recall help? Memory \& Cognition, 44, 1183-1191.

Jeannerod, M. (2006). Motor cognition: What actions tell the self. Oxford: University Press.

Johnson, J. G., \& Raab, M. (2003). Take the first: Option-generation and resulting 
choices. Organizational Behavior and Human Decision Processes, 91(2), 215229. https://doi.org/10.1016/S0749-5978(03)00027-X

Johnson-Glenberg, M. C., Megowan-Romanowicz, C., Birchfield, D. A., \& SavioRamos, C. (2016). Effects of embodied learning and digital platform on the retention of physics content: Centripetal force. Frontiers in Psychology, 7(NOV), 1-22. https://doi.org/10.3389/fpsyg.2016.01819

Jostmann, N. B., Lakens, D., \& Schubert, T. W. (2009). Weight as an embodiment of importance. Psychological Science, 20(9), 1169-1174.

Kalicinski, M., \& Lobinger, B. (2013). Benefits of motor and exercise imagery for older adults. Journal of Imagery Research in Sport and Physical Activity, 8(1), 1-15. DOI: $10.1515 /$ jirspa-2012-0003

Klingberg, T. (2010). Training and plasticity of working memory. Trends in Cognitive Sciences, 14(7), 317-324. https://doi.org/10.1016/j.tics.2010.05.002

Kadosh, R. C. (2014). (Eds.). The stimulated brain: Cognitive Enhancement using noninvasive Brain Stimulation. New York: NY: Elsevier.

Kontra, C., Lyons, D. J., Fischer, S. M., \& Beilock, S. L. (2015). Physical experience enhances science learning. Psychological Science, 26(6), 737-749. https://doi.org/10.1177/0956797615569355

Kouchaki, M., Gino, F., \& Jami, A. (2014). The burden of guilt: Heavy backpacks, light snacks, and enhanced morality. Journal of Experimental Psychology: General, 143(1), 414-424. https://doi.org/10.1037/a0031769

Lachman, R., Lachman, J. L., \& Butterfield, E. C. (1979). Cognitive psychology and information processing. Hillsdale, N. J.: Erlbaum Publishers. 
Lakoff, G., \& Johnson, M. (2003). Metaphors we live by. Chicago: The University of Chicago Press.

Leder, H., Bär, S., \& Topolinski, S. (2012). Covert painting simulations influence aesthetic appreciation of artworks. Psychological Science, 23(12), 1479-1481.

Lillard, A. S. (2005). Montessori: The science behind the genius. Oxford: University Press.

Lindgren, R., Tscholl, M., Wang, S., \& Johnson, E. (2016). Enhancing learning and engagement through embodied interaction within a mixed reality simulation. Computers and Education, 95, 174-187. https://doi.org/10.1016/j.compedu.2016.01.001

Loeffler, J., Raab, M., \& Cañal-Bruland, R. (2016). A lifespan perspective on embodied cognition. Frontiers in Psychology, 7, 845. http://doi.org/10.3389/fpsyg.2016.00845

McMorris, T. (2016). Exercise-cognition interaction: Neuroscience perspectives. (1st Edition). San Diego: Academic Press.

Michalak, J., Burg, J. \&, Heidenreich, T. (2012). Don't forget your body: mindfulness, embodiment, and the treatment of depression. Clinical Psychology \& Psychotherapy, 21, 519-524.

Michalak, J., Troje, N. F., \& Heidenreich, T. (2010). Embodied effects of mindfulnessbased cognitive therapy. Journal of Psychosomatic Research, 68(3), 312-313. https://doi.org/10.1016/j.jpsychores.2010.01.004

Miyake, A., \& Friedman, N. P. (2012). The nature and organisation of individual differences in executive functions: Four general conclusions. Current Directions in Psychological Science, 21(1), 8-14. https://doi.org/10.1177/0963721411429458 
Moreau, D. (2015). Brains and brawn: Complex motor activities to maximize cognitive enhancement. Educational Psychology Review, 27(3), 475-482. https://doi.org/10.1007/s10648-015-9323-5

Moreau, D., Kirk, I. J., \& Waldie, K. E. (2016). Seven pervasive statistical flaws in cognitive training interventions. Frontiers in Human Neuroscience, 10(April), 117. https://doi.org/10.3389/fnhum.2016.00153

Moreau, D., Morrison, A. B., \& Conway, A. R. A. (2015). An ecological approach to cognitive enhancement: Complex motor training. Acta Psychologica, 157, 44-55. https://doi.org/10.1016/j.actpsy.2015.02.007

Munzert, J., Lorey, B., \& Zentgraf, K. (2009). Cognitive motor processes: The role of motor imagery in the study of motor representations. Brain Research Reviews, 60, 306-326.

Nyberg, L., Sandblom, J., Jones, S., Neely, A. S., Petersson, K. M., Ingvar, M., \& Backman, L. (2003). Neural correlates of training-related memory improvement in adulthood and aging. Proceedings of the National Academy of Sciences, 100(23), 13728-13733. https://doi.org/10.1073/pnas.1735487100

Öttl, B., Dudschig, C., \& Kaup, B. (2017). Forming associations between language and sensorimotor traces during novel word learning. Language and Cognition, 9(1), 156-171. https://doi.org/10.1017/langcog.2016.5

Pesce, C., \& Ben-Soussan, T. D. (2016). “Cogito ergo sum” or "ambulo ergo sum”? New perspectives in developmental exercise and cognition research. In T. McMorris (Ed.), Exercise-Cognition Interaction (pp. 251-282). San Diego: Academic Press. 
Pezzulo, G., Barsalou, L. W., Cangelosi, A., Fischer, M. H., McRae, K., \& Spivey, M. J. (2011). The mechanics of embodiment: A dialog on embodiment and computational modeling. Frontiers in Psychology, 2(JAN), 1-21. https://doi.org/10.3389/fpsyg.2011.00005

Potkin, K., Bunney, W., \& García, A. (2012). Sleep improves memory: The effect of sleep on long term memory in early adolescence. PLoS ONE, 7(8), 1-4

Pulvermüller, F. (2005). Brain mechanisms linking language and action. Nature Reviews Neuroscience, 6, 576-582

Pulvermüller, F. (2013). How neurons make meaning: Brain mechanisms for embodied and abstract-symbolic semantics. Trends in Cognitive Sciences, 17(9), 458-470.

Raab, M. (2017). Motor heuristics and embodied choices: how to choose and act. Current Opinion in Psychology, 16, 34-37. https://doi.org/10.1016/j.copsyc.2017.02.029

Raab, M., \& Gigerenzer, G. (2015). The power of simplicity: A fast-and-frugal heuristics approach to performance science. Frontiers in Psychology, 6(OCT), 16. https://doi.org/10.3389/fpsyg.2015.01672

Raab, M., \& Laborde, S. (2011). When to blink and when to think: Preference for intuitive decisions results in faster and better tactical choices. Research Quarterly for Exercise and Sport, 82(1), 89-98. https://doi.org/10.1080/02701367.2011.10599725

Rizzolatti, G., \& Craighero, L. (2004). The mirror-neuron system. Annual Review of Neuroscience, 27(1), 169-192. https://doi.org/10.1146/annurev.neuro.27.070203.144230

Schaeffner, S., Koch, I., \& Phillipp, A. M. (2018). The role of learning in sensory-motor 
modality switching. Psychological Research, 82, 955-969. doi: 10.1007/s00426017-0872-8.

Schütz-Bosbach, S., \& Prinz, W. (2007). Perceptual resonance: action-induced modulation of perception. TRENDS in Cognitive Science, 11(8), 349-355.

Sidhu, D. M. \& Pexman, P. M. (2016). Is moving more memorable than proving? Effects of embodiment and imagined enactment on verb memory. Front. Psychol. 7: 1010. doi: 10.3389/fpsyg.2016.01010

Skulmowski, A., \& Rey, G. D. (2017). Bodily effort enhances learning and metacognition: Investigating the relation between physical effort and cognition using dual-process models of embodiment. Advances in Cognitive Psychology, 13(1), 3-10. https://doi.org/10.5709/acp-0202-9

Slepian, M. L., Masicampo, E. J., Toosi, N. R., \& Ambady, N. (2012). The physical burdens of secrecy. Journal of Experimental Psychology: General, 141(4), 619624. https://doi.org/10.1037/a0027598

Söderqvist, S., \& Nutley, S. B. (2017). Are measures of transfer effects missing the target? Journal of Cognitive Enhancement, 1-5. https://doi.org/10.1007/s41465017-0048-x

Topolinski, S. (2010). Moving the eye of the beholder: Motor components in vision determine aesthetic preference. Psychological Science, 21(9), 1220-1224.

Topolinski, S. \& Strack, F. (2015). Routes to embodiment. Frontiers in Psychology, 6. 10.3389/fpsyg.2015.00940/full

Trevisan, P., Sedeño, L., Birba, A., Ibáñez, A., \& García, A. M. (2017). A moving story: Whole-body motor training selectively improves the appraisal of action meanings 
in naturalistic narratives. Scientific Reports, 7(1), 12538.

https://doi.org/10.1038/s41598-017-12928-w

van Dam, W. O., Rueschemeyer, S. A., Bekkering, H., \& Lindemann, O. (2013).

Embodied grounding of memory: Toward the effects of motor execution on memory consolidation. Quarterly Journal of Experimental Psychology, 66(12), 2310-2328. https://doi.org/10.1080/17470218.2013.777084

Verburgh L, Scherder EJ, van Lange PA, Oosterlaan J. Executive functioning in highly talented soccer players. PloS ONE. 2014;9(3).

Vestberg, T., Gustafson, R., Maurex, L., Ingvar, M., \& Petrovic, P. (2012). Executive functions predict the success of top-soccer players. PLoS ONE, 7(4).

Wegwarth, O., Gaissmaier, W., \& Gigerenzer, G. (2009). Smart strategies for doctors and doctors-in-training: Heuristics in medicine. Medical Education, 43(8), 721728. https://doi.org/10.1111/j.1365-2923.2009.03359.x

Weisberg, S. M., \& Newcombe, N. S. (2017). Embodied cognition and STEM learning: Overview of a topical collection. Cognitive Research: Principles and Implications, 2:38. DOI 10.1186/s41235-017-0071-6

Werner, K., \& Raab, M. (2013). Moving to solution. Experimental Psychology, 60, 403409.

Woods, A. J., Philbeck, J. W. \& Danoff, J. V. (2009). The various perceptions of distance: An alternative view of how effort affects distance judgments Journal of Experimental Psychology: Human Perception and Performance, 35, 1104-1117.

Wriessnegger, S., Steyrl, D., Koschutnig, K., \& Müller-Putz, G. (2014). Short time sports exercise boosts motor imagery patterns: Implications of mental practice in 
rehabilitation programs. Frontiers in Human Neuroscience, 8(469), 1-9.

Xie, L., Kang, H., Xu, Q., Chen, M. J., Liao, Y., Thiyagarajan, M., . . Nedergaard, M. (2013). Sleep drives metabolite clearance from the adult brain. Science, $342(6156), 373-377$. 


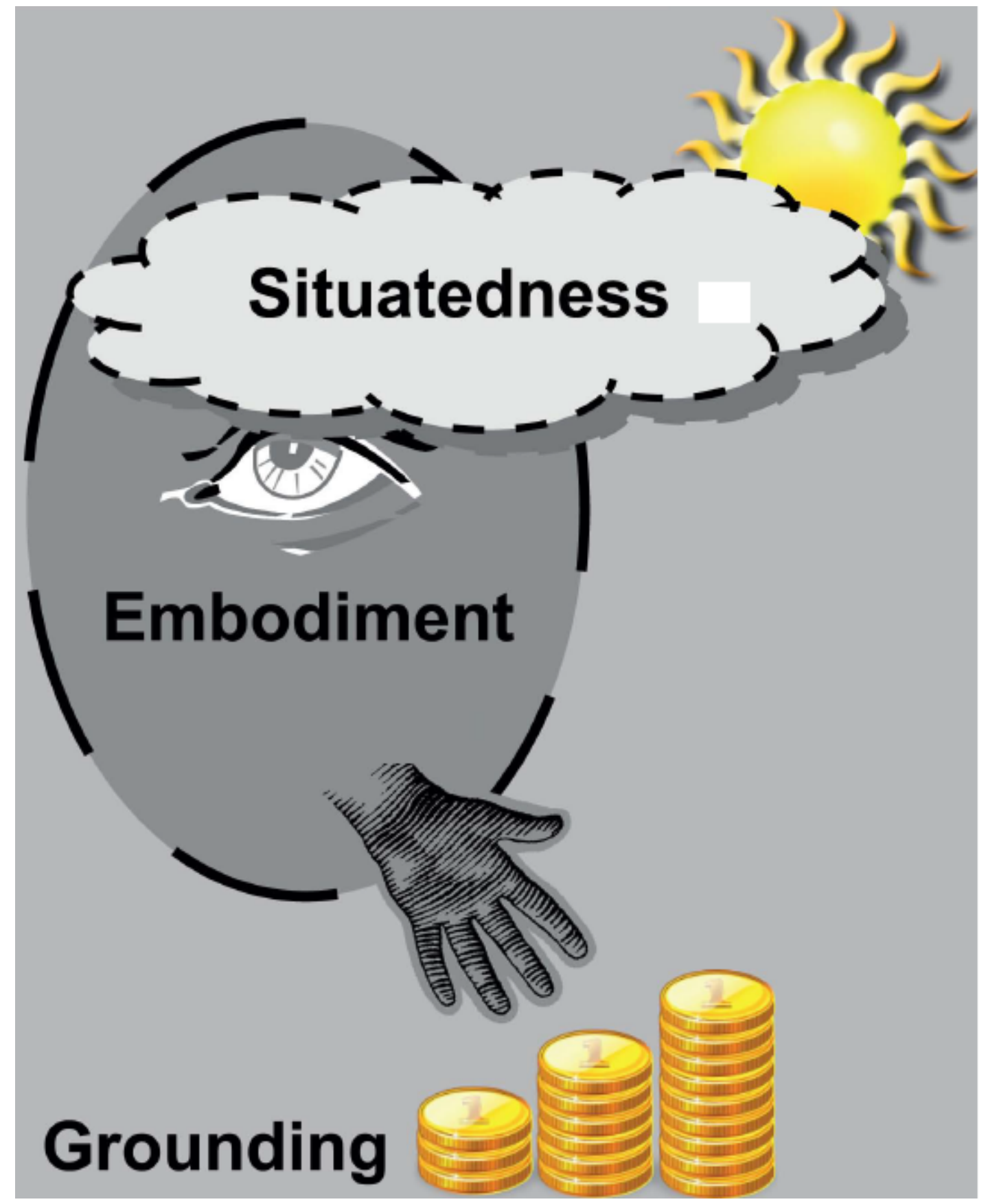

Figure 1. The hierarchical relationship between grounding, embodiment and situated cognition (modified from Fischer \& Brugger, 2011). See text for details. 


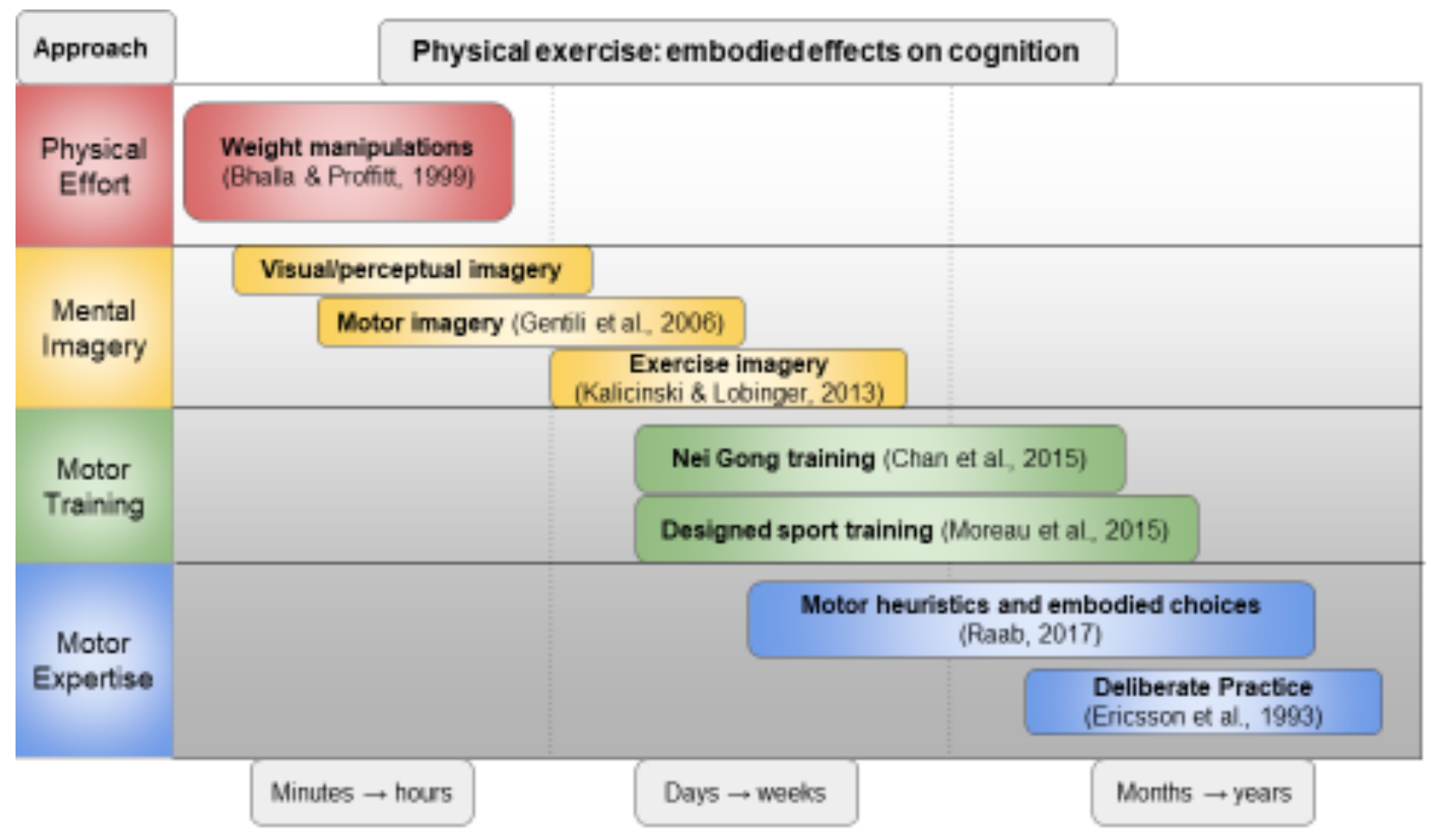

Figure 2. An overview of effects of embodied exercise regimes on enhanced cognition at different time scales. See text for details. 


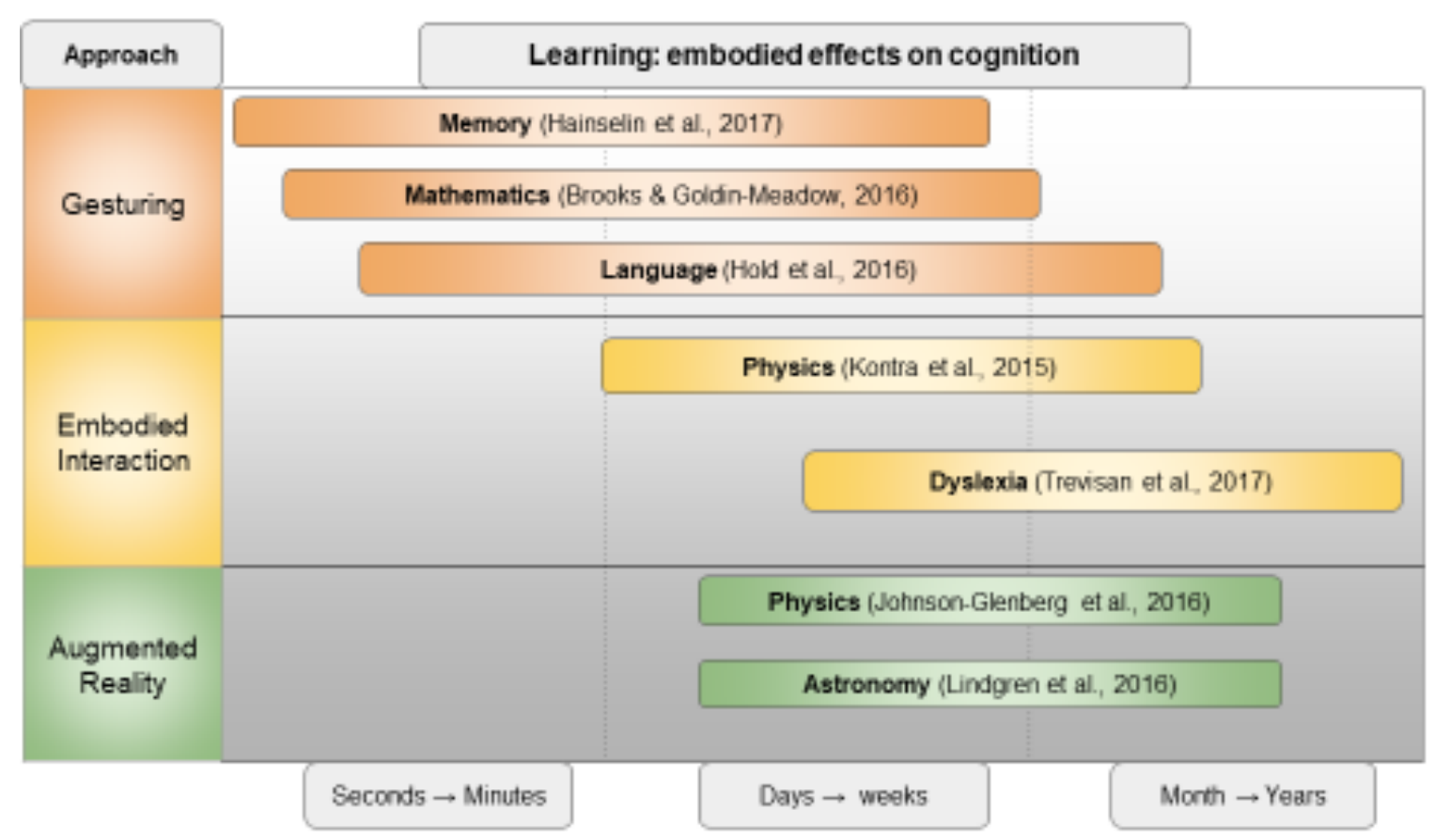

Figure 3. An overview of effects of embodied learning regimes on enhanced cognition at different time scales. See text for details. 